\title{
Stepwise Treatment for Abnormally Invasive Placenta with Placenta Previa
}

\author{
Seiji Sumigama, MD, PhD ${ }^{1,2}$ Tomomi Kotani, MD, $\mathrm{PhD}^{1}$ Hiromi Hayakawa, MD, $\mathrm{PhD}^{3}$
}

1 Department of Obstetrics and Gynecology, Nagoya University Graduate School of Medicine, Nagoya, Japan

2 Department of International Medical Education, Nagoya University Graduate School of Medicine, Nagoya, Japan

${ }^{3}$ Department of Obstetrics, Aichi Children's Health and Medical Center, Obu, Aichi, Japan
Address for correspondence Seiji Sumigama, MD, PhD, Department of Obstetrics and Gynecology, Nagoya University Graduate School of Medicine, 65 Tsurumai-cho, Showa-ku, Nagoya 466-8550, Japan (e-mail: sumigama@med.nagoya-u.ac.jp).

\section{Surg J (NY) 2021;7(suppl S1):S20-S27.}
Abstract
Keywords
- placenta accreta spectrum
- abnormally invasive placenta
- placenta previa
- modified cesarean hysterectomy
- stepwise treatment
- uterine arterial embolization
- catheter occlusion

Placenta accreta spectrum (PAS) disorder often causes a large amount of intraoperative bleeding in a short period which makes maternal circulation unstable and threatens life. As a countermeasure, two-stage surgery combined with selective uterine arterial embolization (UAE), named "stepwise treatment" was introduced in 2003. At a cesarean section (CS), only the baby is delivered and the placenta is left in situ. The transcatheter angiographic UAE is performed on the operation day, followed by the total hysterectomy on 5 to 7 days after CS. The difficulty in the operative procedures for hysterectomy and the amount of bleeding can be reduced by the added effect of the blood flow interruption by UAE and the uterine involution. Although there are not many indication cases, this is the prudent operation that should be considered for the most severe PAS case such as total placenta increta/percreta with placenta previa.

In this article, the practical procedures and tips of stepwise treatment are described.

\section{Surgical Steps}

1. Cesarean section: Laparotomy to delivery. $\downarrow$

2. Cesarean section: Assessment of the difficulty of hysterectomy, decision on operative policy. $\downarrow$

3. Cesarean section: Placenta preservation, hysterotomy closure, and tube/ovarian ligament ligation. $\downarrow$

4. Selective uterine arterial embolization (on the operative day of CS): Angiography and embolization. $\downarrow$

published online September 30, 2021
DOI https://doi.org/ $10.1055 / \mathrm{s}-0041-1728748$ ISSN 2378-5128.
5. Total hysterectomy (on 5 to 7 days after CS): Preparation and laparotomy.

$\downarrow$

6. Total hysterectomy: Round ligament, ovarian ligament and broad ligament.

$\downarrow$

7. Total hysterectomy: Uterosacral ligament. $\downarrow$

8. Total hysterectomy: Lateral treatment and lower uterine segment bypass. $\downarrow$

9. Total hysterectomy: Cardinal ligament. $\downarrow$

10. Total hysterectomy: Vaginal canal incision and dissection of uterine-bladder invasion. (c) 2021. The Author(s).

This is an open access article published by Thieme under the terms of the Creative Commons Attribution-NonDerivative-NonCommercial-License, permitting copying and reproduction so long as the original work is given appropriate credit. Contents may not be used for commercial purposes, or adapted, remixed, transformed or built upon. (https://creativecommons.org/ licenses/by-nc-nd/4.0/)

Thieme Medical Publishers, Inc., 333 Seventh Avenue, 18th Floor, New York, NY 10001, USA 


\section{Operative Indication}

We performed this planned stepwise treatment for the first time in 2003. ${ }^{1}$ This protocol features two-step operations incorporating the selective uterine arterial embolization (UAE) with the view to prevent massive short-time bleeding which occurs characteristically in obstetrical surgeries. By the added effect of blood flow interruption by the arterial embolization and involution of the uterus, the cases treated by this method successfully reduced operative bleeding compared with the cases treated with other treatments. ${ }^{2}$ The essential requirement for this treatment is total placenta accreta spectrum (PAS) in which the entire placental bed firmly attaches to the uterine myometrium resulting in no separating hemorrhage during the waiting period between the cesarean section (CS) and the hysterectomy. By contrast, in the case of partial PAS in which certain part of placenta begins to detach and bleeding occurs after childbirth, the operator is forced to perform cesarean hysterectomy at a time (-Fig. 1). On the basis of total PAS, the severe form of PAS is a suitable indication, i.e., placenta increta/percreta, also known as abnormally invasive placenta (AIP) in which placenta invades into the uterine wall and sometimes reaches surrounding organs with the marked proliferation of abnormal blood vessels on the uterine surface. In addition, PAS complicated with placenta previa is also distressing for surgeons because the lower part of the uterus is larger than fundus which worsens the lateral and posterior view of the operative field and tightens the clearance between the uterus and pelvis wall that leads to the difficulty in dissecting the lower part of the uterus. Thus, AIP with placenta previa can be recognized as the most severe phenotype and the most suitable indication for stepwise treatment.

\section{Preoperative Evaluation and Preparation}

To distinguish total and partial accreta to make a treatment plan, the depth and expanse of adhesion/invasion are evaluated preoperatively by ultrasonography and magnetic resonance imaging (MRI): visualization of placental lacunae, lack of a sonolucent zone, interruption of the sonolucent line appearing at the posterior bladder wall-uterine interface on B mode ultrasonography, visualization of turbulent lacunae flow on Doppler ultrasonography, indistinct border of the myometrium, placental bulging, and heterogeneity on MRI are characteristic observations in PAS. When the bladder dome is lifted to the uterine body in the sagittal section, the bladder dissection is expected to be difficult.

Autologous blood should be collected as much as possible. As long as the clinical course is stable, the operation is scheduled at 36 to 37 weeks, whereas, if the clinical course is not stable, e.g., alarm bleeding or preterm labor is observed, the elective operation at earlier than 36 weeks is acceptable to avoid emergency surgery.

\section{Operative Procedures}

\section{Cesarean Section}

Step 1. Laparotomy and delivery

The lithotomy position or the open leg position is applied to perform the transvaginal examination and to recognize transvaginal bleeding. The general anesthesia is applied with two infusion roots accompanied by heating devices in preparation for the massive fluid replacement and transfusion against serious bleeding. Though the benefit is still controversial, we insert ureteral catheters to prevent ureteral damage. The presence of invasion of the placenta reaching

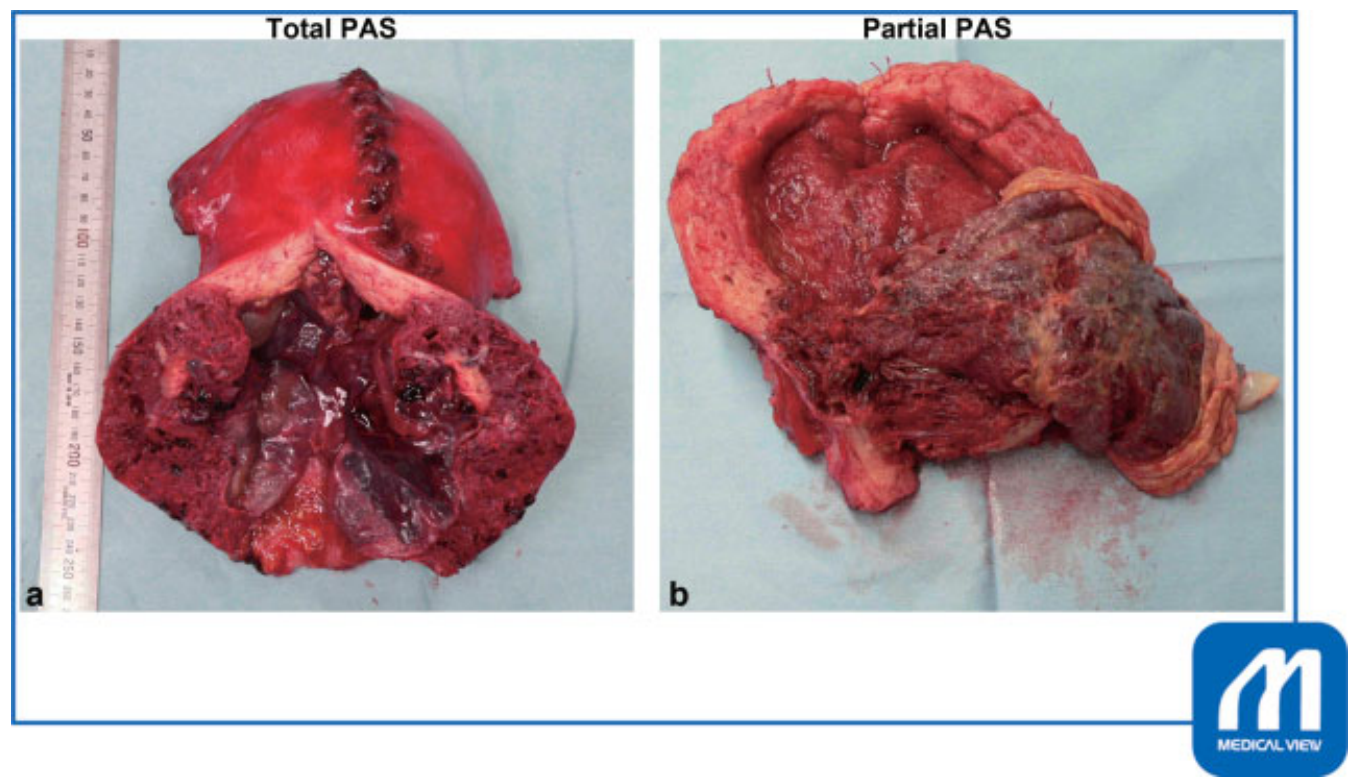

Fig. 1 Total and partial PAS. (a) Total PAS (b) Partial PAS. (a) The entire placenta is unified with the uterus in total PAS. (b) Only a part of the placenta attaches the uterus in partial PAS. (Reproduced with permission of Sumigama S, Kotani T, Hayakawa H. Stepwise treatment. In: Hiramatsu Y, Konishi I, Sakuragi N, Takeda S, eds. Surgery for Pregnancy with Placenta Previa and Placenta Accrete: Careful Preparation and Critical Management. OGS NOW, No.9. (Japanese). Tokyo: Medical View; 2012:134-143. Copyright@ Medical View). PAS, placenta accreta spectrum. 


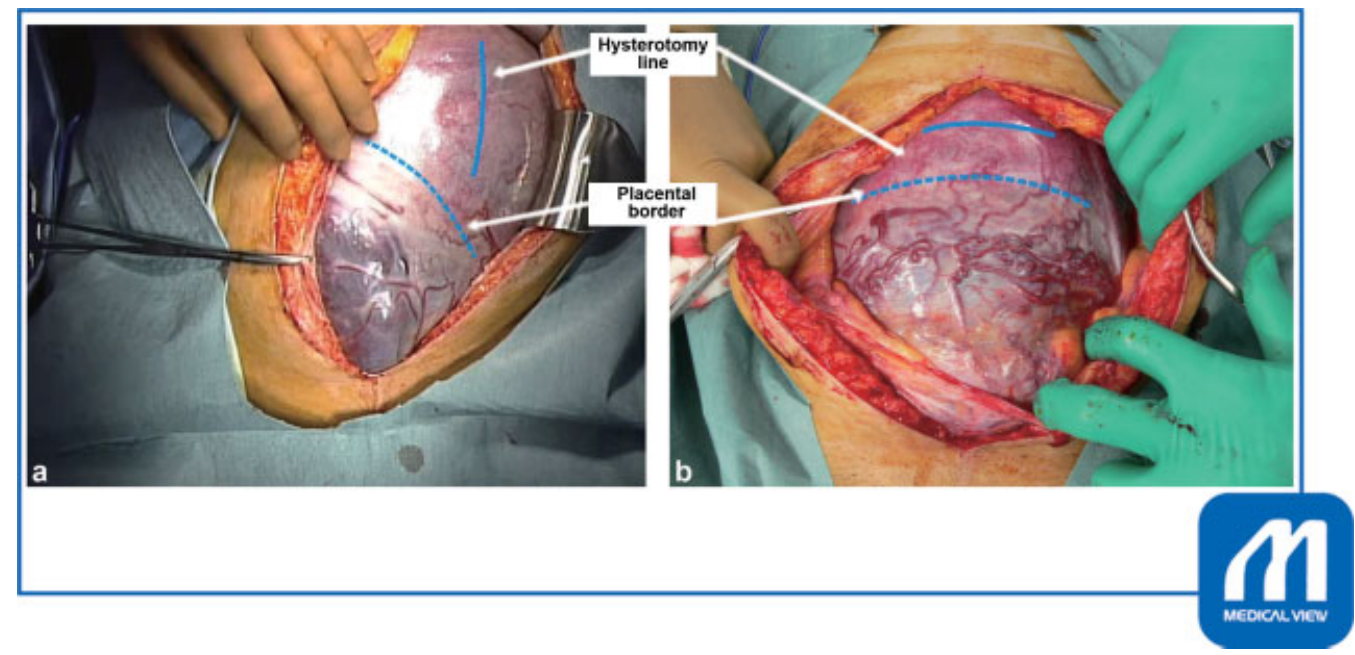

Fig. 2 The hysterotomy line (solid line) in the cesarean section should be placed with a sufficient margin from the border of the placenta (dotted line) not to cut into the placenta. (a) The incision was made at the uterine body longitudinally, slightly left sided because the placenta was located at the anterior to the right lateral wall of the lower segment. (b) The incision was made at the fundus transversely because the placenta was located at the anterior body. (Reproduced with permission of Sumigama S, Kotani T, Hayakawa H. Stepwise treatment. In: Hiramatsu Y, Konishi I, Sakuragi N, Takeda S, eds. Surgery for Pregnancy with Placenta Previa and Placenta Accrete: Careful Preparation and Critical Management. OGS NOW, No.9. (Japanese). Tokyo: Medical View; 2012:134-143. Copyright@ Medical View).

the inner surface of the bladder can be checked by the cystoscope simultaneously.

The abdominal wall is longitudinally incised. The hysterotomy line should be placed away from the placental border according to the preoperative observation of placental location by ultrasonography/MRI. If applicable, intraoperative ultrasonography can directly confirm the placental attachment site. Since a hysterectomy is premised, the hysterotomy can be a vertical or horizontal incision of the uterine body. Care must be taken not to cut in the placenta (-Fig. 2).

Step 2. Assessment and decision on operative policy

After childbirth, placenta invasion and suspected difficulty level of the hysterectomy based on intraoperative findings are assessed to decide whether stepwise treatment can be performed as planned based on the following three factors:

(1) Absence of the placental separation: to clarify there are no signs of bleeding from the uterine cavity that comes along with placental separation after administration of uterotonics. We administer uterotonics to let the placenta separate as long as it does. Stepwise treatment must confirm that placental attachment is firm against the uterine contraction.

(2) The existence of aberrant blood vessels on the vesicouterine pouch: this finding implies that the mobilization of the bladder will be difficult and will cause massive hemorrhage.

(3) The bidigital palpation of the vesicouterine pouch to anterior vaginal fornix distance (-Fig. 3a), Douglas fossa

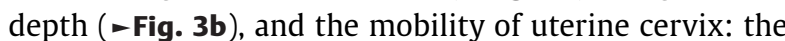
long "a" "b" distance and/or the poor mobility of the cervix imply lots of procedures with difficulty will be needed to free the uterine cervix from the pelvic floor. Thus, these are applicable indicators for the stepwise treatment.

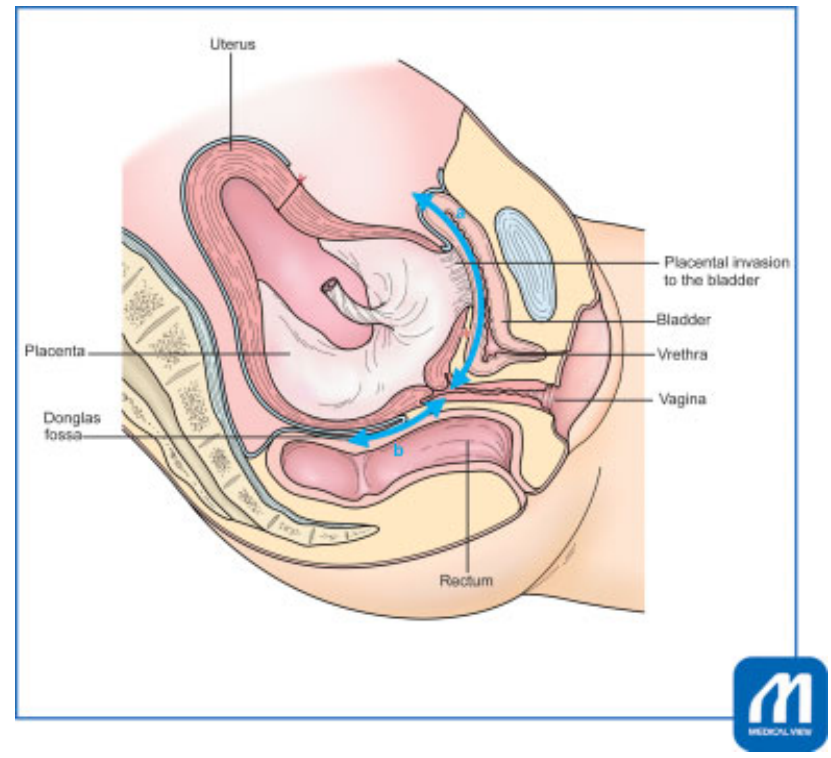

Fig. 3 Estimation of the difficulty of hysterectomy by the bidigital palpation. The distance " $a$ " between the vesicouterine pouch to the anterior vaginal fornix, the distance " $b$ " between Douglas fossa and posterior vaginal fornix, and the mobility of uterus are assessed. Long " $a$ " and/or " $b$ " distance and poor mobility imply a high degree of difficulty is needed for the hysterectomy. The asterisk “*” indicates the site for creating a cervical bypass before the dissection of the invasion between the uterus and bladder. (Reproduced with permission of Sumigama S, Kotani T, Hayakawa H. Stepwise treatment. In: Hiramatsu Y, Konishi I, Sakuragi N, Takeda S, eds. Surgery for Pregnancy with Placenta Previa and Placenta Accrete: Careful Preparation and Critical Management. OGS NOW, No.9. (Japanese). Tokyo: Medical View; 2012:134-143. Copyright@ Medical View). Uterus placenta Douglas fossa rectum placental invasion to the bladder urethra vagina.

Step 3. Placenta preservation, hysterotomy closure, tube and ovarian ligament ligation

The placenta is left in situ and the uterine incision is closed. In our initial few cases, a drain tube was inserted into 
the uterine cavity to monitor bleeding and the intrauterine infection, but neither bleeding nor infection was sustained. The tube and ovarian ligaments are clamped and suture ligated to block the blood flow to the uterine through the ovarian artery. No incision in the tube and ovarian ligaments should be made to avoid the possibility of bleeding from the stump while waiting for hysterectomy. This procedure can also prevent ovarian failure by avoiding embolic materials flown into the ovary via the ascending branch of the uterine artery during the arterial embolization. ${ }^{3}$ After the confirmation of no vaginal bleeding, the abdomen is closed.

\section{Selective Uterine Arterial Embolization}

\section{Step 4. Angiography and embolization}

UAE is performed immediately after the CS. The pelvic angiography identifies the feeding arteries to the uterus and placenta which includes the uterine, vesical and internal pudendal artery from the internal iliac artery. Note that not only branches from the internal iliac artery, but also branches from the external iliac artery, e.g., inferior epigastric artery and its branches to round ligament and pubis, and external pudendal artery, supply blood flow to the uterus, placenta, and involved bladder in the pregnant uterus complicated with PAS. ${ }^{4,5}$ Then, embolization is performed using platinum coils for medium-sized arteries and gelatin sponges particles for peripheral arteries are used as the embolic material. In the selective UAE, embolic materials can block the blood flow at the more peripheral level than the balloon catheter occlusion. This is the advantage of UAE over the balloon catheter occlusion which cannot block the blood flow through the peripheral collateral arterial connection.

Fever, lower limb ischemia, pelvic viscera necrosis, and neuropathy were reported as complications of embolization. In our cases, fever and increased C-reactive protein were observed; however, no causative pathogens were identified in the fluid from the drain tube placed in the uterine cavity.

\section{Total Hysterectomy}

\section{Step 5. Preparation and laparotomy}

A hysterectomy is scheduled on no later than 14 days, usually on 5 to 7 days after the UAE before the recanalization of arteries occurs due to the meltdown of gelatin sponge particles. The uterine involution also leads to the reduction of uterine blood flow, and the maternal general condition can be stabilized if the massive hemorrhage caused anemia and circulatory insufficiency after the CS (-Fig. 4). Because the placenta occupies the cervix in previa complicated cases, not a supra hysterectomy but a total hysterectomy is performed.

The general anesthesia is inducted and multiple infusion routes with blood transfusion heating circuits are prepared. The abdominal wall is longitudinally incised. An incision up to the umbilicus is often required.

\section{Tips and Warnings}

\section{“Traction by the Assistant"}

The lower part of the uterus is larger than fundus and shows a pear shape which worsens the lateral and pos- terior view of the operative field and tightens the clearance between the uterus and pelvis wall that leads to the difficulty in procedures of the lower uterine segment. The total hysterectomy of AIP with placenta previa can be said as the process in which a large uterus buried in the pelvis being dug up while its surrounding tissues being peeled off little by little. In this process, the assistant plays an important role in presenting the dissecting site by grasping the uterine body with forceps, elevating it, and exerting the traction toward the opposite side stronger than in an orthodox hysterectomy. Loose connective tissues around the lower uterine segment become tightened to be peeled off with blunt dissection or an electric scalpel and vessels become exposed to be cut and ligated. By repeating these processes, the uterine cervix buried in the pelvis is gradually excavated (-Fig. 5)

\section{Tips and Warnings}

\section{"Start from the Small Things First" Policy}

When the operator faces difficulties in dissecting tissues at a site, it is better not to overdo there but to do another step at another site first because the situation may change in a later phase. Thus, the following steps 6 to 8 can be done in order of increasing difficulty case by case. Especially, the procedures of dissecting the invasion between the anterior uterine wall and the bladder are the most annoying because there is a bunch of vascular formation at the vesicouterine pouch and the operator tends to lose the orientation in layers because uterine and bladder wall becomes thin and normal layers are broken by placental invasion. Thus, it is recommended to put off the dissection of the uterovesical invasion till the last phase and to proceed to insect the parametrium and vaginal duct first. This procedure is introduced by Pelosi and Pelosi as "modified cesarean hysterectomy."

Step 6. Round ligament, ovarian ligament, and broad ligament

The round ligament is often extended by the existence of the underlying placenta; thus, it should be clamped and cut at the pelvic side to keep enough margin of the stump of the uterine side to be reliably suture ligated. The incision is made in the anterior leaflet of the broad ligament peritoneum from the stump of the round ligament toward the tube/ovarian ligament which was ligated during the CS, and the tube/ovarian ligament is clamped, cut, and suture ligated. The anterior leaflet of the peritoneum is also cut toward the midline to open the vesicouterine pouch. Devascularization of the pouch is performed carefully, and the bladder is 


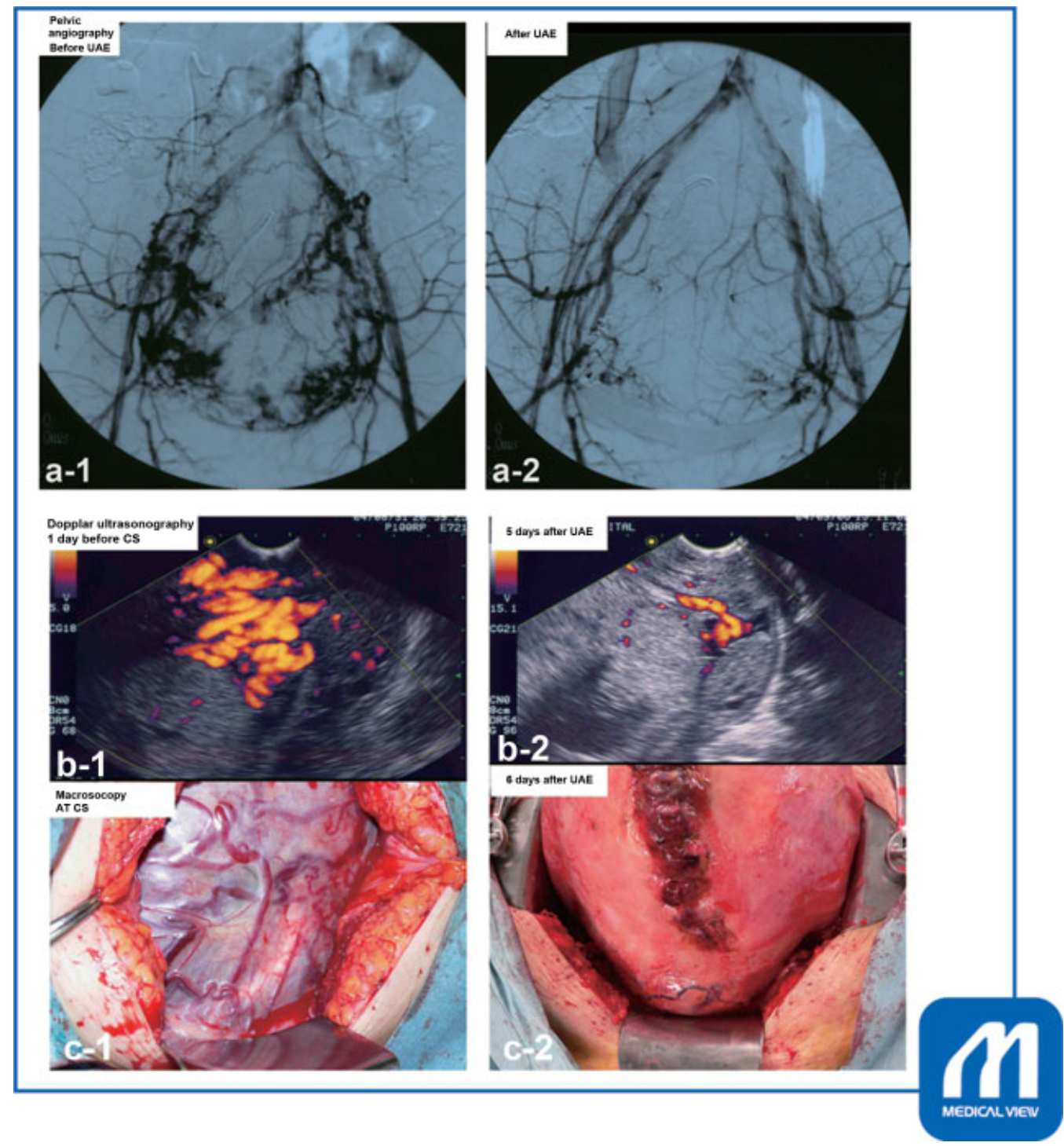

Fig. 4 Changes in the uterine blood flow before and after UAE. (a) Pelvic angiography. (a-1) before, (a-2) after UAE. Before the embolization, the angiography showed marked blood flow coincides with the uterus and placenta (a-1), which disappeared after the embolization (a-2). (b) Doppler ultrasonography (b-1) before CS, (b-2) 5 days after UAE Doppler ultrasonography showed the marked blood flow signal before the embolization (b-1, before the cesarean section performed), whereas the signal remarkably reduced 5 days after the embolization (b-2). (c) Macroscopy (c-1) At CS, (c-2) 6 days after UAE macroscopic examination revealed abnormal blood vessels on the surface of the uterus (c-1, at the cesarean section), whereas the vessels remarkably shrank 6 days after the embolization (c-2, at the hysterectomy). (Reproduced with permission of Sumigama S, Kotani T, Hayakawa H. Stepwise treatment. In: Hiramatsu Y, Konishi I, Sakuragi N, Takeda S, eds. Surgery for Pregnancy with Placenta Previa and Placenta Accrete: Careful Preparation and Critical Management. OGS NOW, No.9. (Japanese). Tokyo: Medical View; 2012:134-143. Copyright@ Medical View). UAE, uterine arterial embolization.

mobilized caudally till reaching the firm attaching site to the lower uterine segment. Injection of 100 to $200 \mathrm{~mL}$ of normal saline solution into the bladder makes it easier to identify the border between the bladder and the uterus.

\section{Step 7. Uterosacral ligament}

An incision is made in the posterior leaflet of the peritoneum vertically toward the uterosacral ligament. Adhesion to the posterior wall is often less severe than that of the anterior wall and there must be less aberrant blood vessels. Instead, the operative view is poor because of the enlargement of the lower part of the uterus including the placenta. With the intensive elevation of the uterus by the assistant, the uterosacral ligament becomes accessible to be clamped, cut, and suture ligated.

Step 8. Lateral treatment and lower uterine segment bypass

When the assistant elevates the uterus and exerts the traction toward the opposite side intensively, loose connective tissues around the cardinal ligament become tightened which makes it easier to dissect it with blunt or sharp dissection. After the dissection of connective tissue, the uterine artery becomes exposed and the ureter comes away from the uterus. To reduce the risk of ureter injury, the ureter is identified by palpating the catheter inserted and 


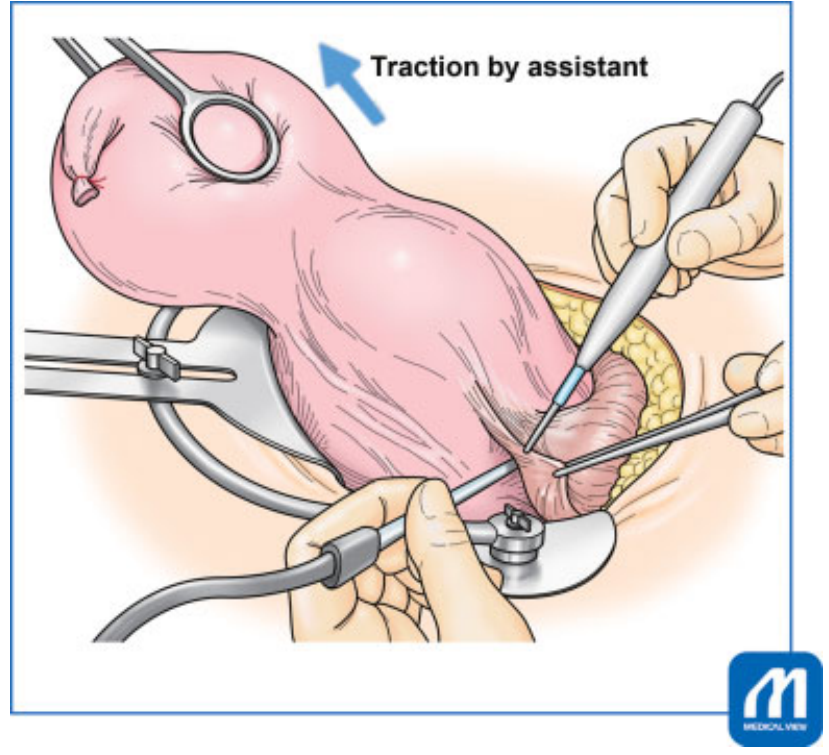

Fig. 5 Dissection procedures around the lower uterine segment The strong uterine traction to the opposite side reveals and tightens the connective tissues and vessels around the uterus to be easily dissected or devascularized. (Reproduced with permission of Sumigama S,

Kotani T, Hayakawa H. Stepwise treatment. In: Hiramatsu Y, Konishi I, Sakuragi N, Takeda S, eds. Surgery for Pregnancy with Placenta Previa and Placenta Accrete: Careful Preparation and Critical Management. OGS NOW, No.9. (Japanese). Tokyo: Medical View; 2012:134-143. Copyright@ Medical View).

pushed away further laterally. Then, the left and right fingers are inserted into the loose connective tissue at the paravesical spaces bilaterally toward the midline to create a bypass passing through side to side below the uterinebladder invasion site (indicated by ${ }^{*}$ in - Fig. 3; - Fig. 6). The lower the bypass positions, the easier to make it because the placenta rarely invades into the lower cervix. By enlarging the bypass caudally, the vaginal canal is exposed.

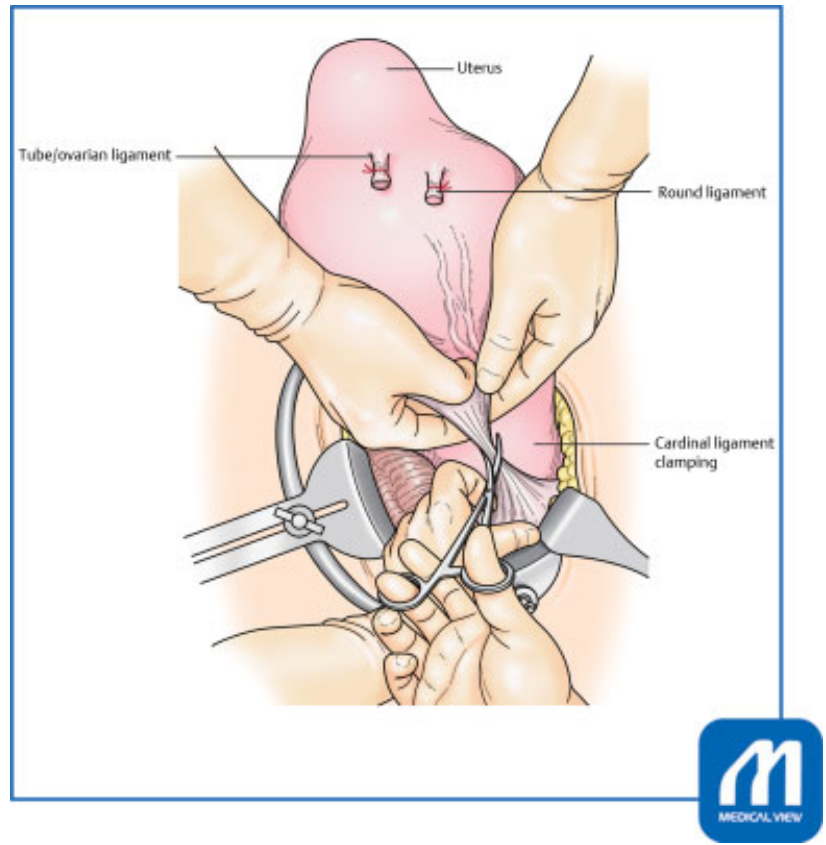

Fig. 7 Dissection of the cardinal ligament The assistant grasps the parametrium with fingers, pulls upward, and elevates the opposite side to present the parametrium so that the operator can easily clamp it. (Reproduced with permission of Sumigama S, Kotani T, Hayakawa H. Stepwise treatment. In: Hiramatsu Y, Konishi I, Sakuragi N, Takeda S, eds. Surgery for Pregnancy with Placenta Previa and Placenta Accrete: Careful Preparation and Critical Management. OGS NOW, No.9. (Japanese). Tokyo: Medical View; 2012:134-143. Copyright $\odot$ Medical View). Tube/ovarian ligament uterus round ligament cardinal ligament clamping.

Step 9. Cardinal ligament

Uterine traction by the assistant is again important when clamping the cardinal ligament. When the uterus is pulled upward and toward the opposite side, the cardinal ligament

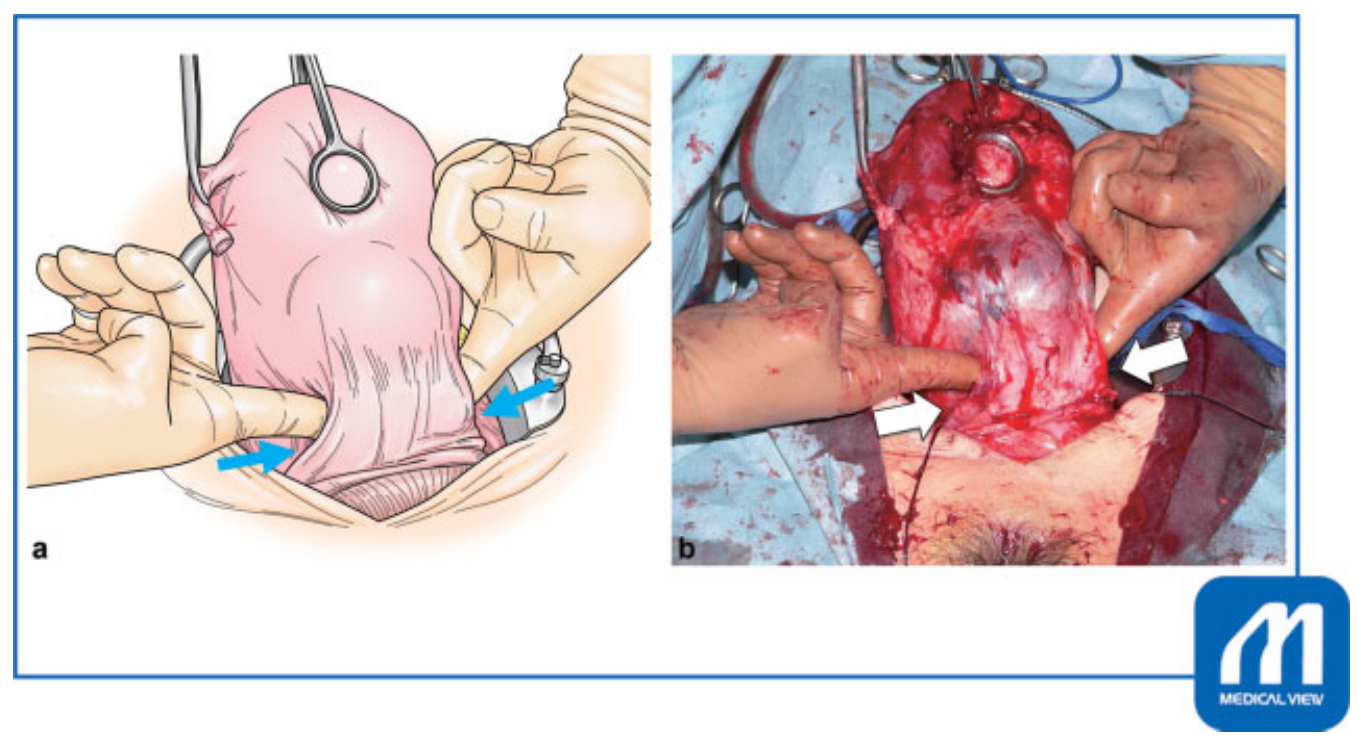

Fig. 6 The bypass is created below the uterine-bladder invasion site. Fingers are inserted at the paravesical space bilaterally. (Reproduced with permission of Sumigama S, Kotani T, Hayakawa H. Stepwise treatment. In: Hiramatsu Y, Konishi I, Sakuragi N, Takeda S, eds. Surgery for Pregnancy with Placenta Previa and Placenta Accrete: Careful Preparation and Critical Management. OGS NOW, No.9. (Japanese). Tokyo: Medical View; 2012:134-143. Copyright@ Medical View). 


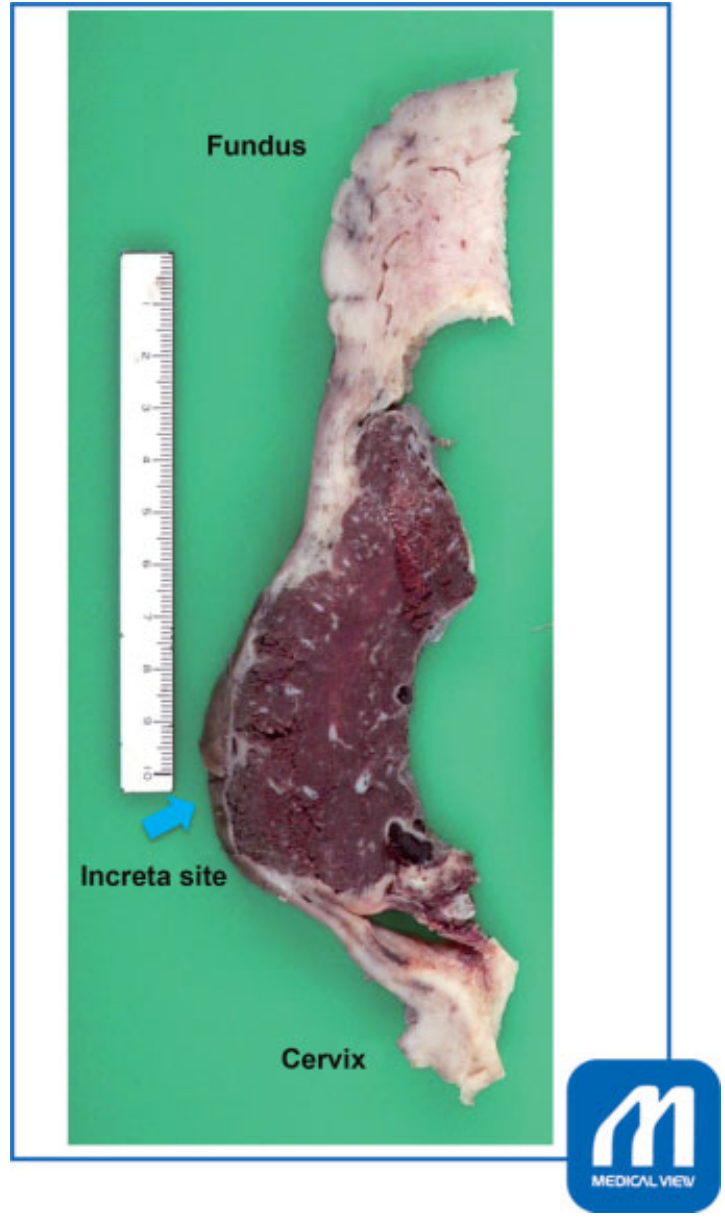

Fig. 8 A formalin-fixed specimen of placenta increta shows the thinning of the myometrium at the placental increta site. Note that deep incision into the fascia reaches the placenta immediately below, causing uncontrolled bleeding. (Reproduced with permission of Sumigama S, Kotani T, Hayakawa H. Stepwise treatment. In: Hiramatsu Y, Konishi I, Sakuragi N, Takeda S, eds. Surgery for Pregnancy with Placenta Previa and Placenta Accrete: Careful Preparation and Critical Management. OGS NOW, No.9. (Japanese). Tokyo: Medical View; 2012:134-143. Copyright $\odot$ Medical View). Fundus increta site cervix.

including the uterine artery is exposed. Forceps that do not easily slip off, such as serrated forceps are placed at the inner side of the secured ureter, and the cardinal ligament including the ascending branch of the uterine artery is clamped, cut, and suture ligated ( - Fig. 7 ). By repeating it at the inner side of the pedicle, the uterine cervix is freed from the cardinal ligament. It is important not to cut into the fascia deeply because the placenta is located directly below the fascia and causes uncontrollable bleeding when cut in (extrafascial hysterectomy, ${ }^{7}$ - Fig. 8).

Step 10. Vaginal canal incision and dissection of uterinebladder invasion

Before cutting in the vaginal canal, the incision line should be marked according to the guide by the assistant's finger inserted at the anterior and posterior vaginal fornix because the pregnant uterus is so soft that it is difficult to palpate the vaginal portion of the cervix correctly from the external side of the canal. Since the invasion between the uterus and the

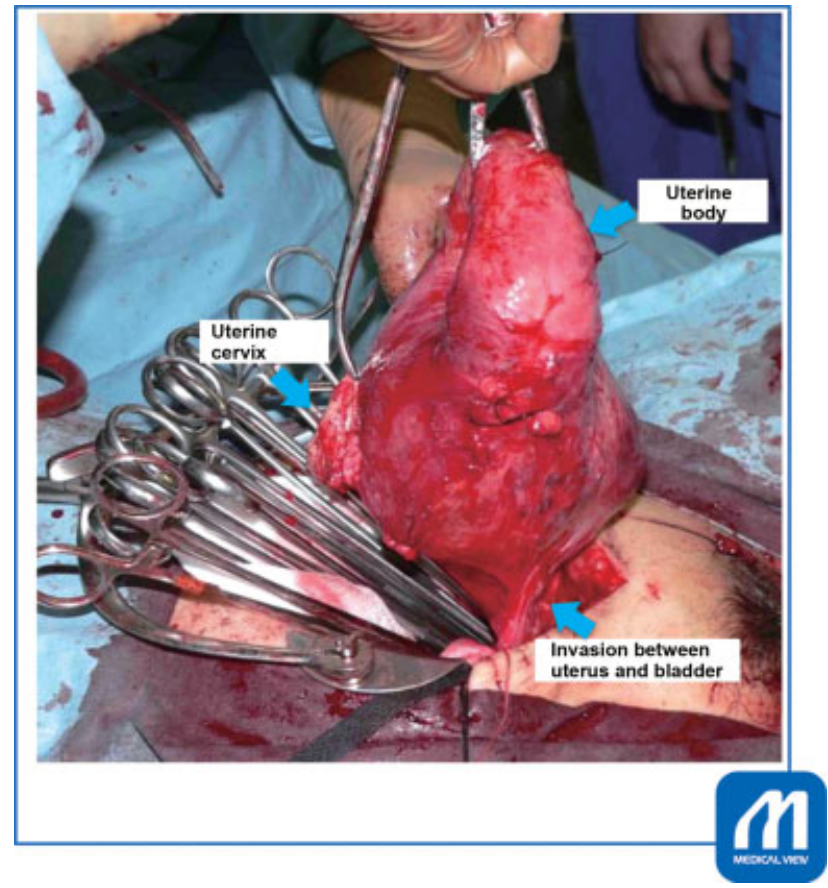

Fig. 9 After the incision of the vaginal canal, the uterus connects only by the adhesions to the bladder. (Reproduced with permission of Sumigama S, Kotani T, Hayakawa H. Stepwise treatment. In: Hiramatsu Y, Konishi I, Sakuragi N, Takeda S, eds. Surgery for Pregnancy with Placenta Previa and Placenta Accrete: Careful Preparation and Critical Management. OGS NOW, No.9. (Japanese). Tokyo: Medical View; 2012:134-143. Copyright $\odot$ Medical View). Uterine cervix uterine body invasion between uterus and bladder.

bladder remains, the vaginal canal is incised from the posterior or lateral wall ( $\mathbf{- F i g . ~ 9 ) . ~ B l e e d i n g ~ f r o m ~ t h e ~ v a g i n a l ~}$ stump is sometimes severe, thus the suture and hemostasis are performed firmly. Lastly, the connecting part between the uterus and the bladder is separated off. If the bladder is broken, the edge of the bladder can be found by putting a finger into the bladder that makes the dissecting line easily identified. The amount of bleeding by the dissection of this invasion site is expected to be minimized at this time because the main blood supplies are already terminated. The thinned bladder wall is sutured layer by layer with absorbable threads. Hemostasis is confirmed and the abdomen is closed.

\section{Conclusion}

Stepwise treatment is one of the surgical options for severe PAS cases such as AIP with placenta previa. The 3-step protocol of CS, arterial embolization, and hysterectomy must be a burden for the patient; however, it has a great advantage that the massive uterine hemorrhage is reduced, and thus operations can be performed under the stable circulatory condition which must contribute to the improvement of maternal outcomes.

\section{Conflict of Interest}

None. 


\section{References}

1 Sumigama S, Kotani T, Hayakawa H. Stepwise treatment. In: Hiramatsu Y, Konishi I, Sakuragi N, Takeda S, eds. OGS NOW No9 Surgery for Pregnancy with Placenta Previa and Placenta Accreta: Careful Preparation and Critical Management. Tokyo: Medical View; 2012:134-143

2 Sumigama S, Itakura A, Ota T, et al. Placenta previa increta/percreta in Japan: a retrospective study of ultrasound findings, management and clinical course. J Obstet Gynaecol Res 2007;33(05):606-611

3 Spies JB, Spector A, Roth AR, Baker CM, Mauro L, Murphy-Skrynarz K. Complications after uterine artery embolization for leiomyomas. Obstet Gynecol 2002;100(5 Pt 1):873-880
4 Badawy SZ, Etman A, Singh M, Murphy K, Mayelli T, Philadelphia $\mathrm{M}$. Uterine artery embolization: the role in obstetrics and gynecology. Clin Imaging 2001;25(04):288-295

5 Cheng YY, Hwang JI, Hung SW, et al. Angiographic embolization for emergent and prophylactic management of obstetric hemorrhage: a four-year experience. J Chin Med Assoc 2003;66(12): 727-734

6 Pelosi MA III, Pelosi MA. Modified cesarean hysterectomy for placenta previa percreta with bladder invasion: retrovesical lower uterine segment bypass. Obstet Gynecol 1999;93(5 Pt 2):830-833

7 Cibula D, Abu-Rustum NR, Benedetti-Panici P, et al. New classification system of radical hysterectomy: emphasis on a threedimensional anatomic template for parametrial resection. Gynecol Oncol 2011;122(02):264-268 